\title{
The risk factors of concomitant intraperitoneal and retroperitoneal hemorrhage in the patients with blunt abdominal trauma
}

\author{
Chun-Yi Wu ${ }^{\dagger}$, Shang-Ju Yang ${ }^{\dagger}$, Chih-Yuan Fu*, Chien-Hung Liao, Shih-Ching Kang, Yu-Pao Hsu, Being-Chuan Lin, \\ Kuo-Ching Yuan and Shang-Yu Wang
}

\begin{abstract}
Introduction: Intraperitoneal and retroperitoneal hemorrhages may occur simultaneously in blunt abdominal trauma (BAT) patients. These patients undergo emergency laparotomies because of concomitant unstable hemodynamics and positive sonographic examination results. However, if the associated retroperitoneal hemorrhage is found intraoperatively and cannot be controlled surgically, then the patients require post-laparotomy transcatheter arterial embolization (TAE). In the current study, we attempted to determine the risk factors for post-laparotomy TAE.
\end{abstract}

Materials and methods: Patients with concomitant BAT and unstable hemodynamic were retrospectively analyzed. The characteristics of the patients who underwent laparotomy or who required post-laparotomy TAE were investigated and compared. The Tile classification system was used to evaluate the pelvic fracture patterns.

Results: Seventy-four patients were enrolled in the study. Fifty-nine (79.7\%) patients underwent laparotomy to treat intra-abdominal hemorrhage, and fifteen (20.3\%) patients underwent additional post-laparotomy TAE because of concomitant retroperitoneal hemorrhage. Pelvic fracture was present in $80.0 \%$ of the post-laparotomy TAE patients. This percentage was significantly greater than that of the laparotomy only patients ( $80.0 \%$ vs. $30.5 \%, p<0.001)$. Furthermore, 30 patients $(40.5 \%, 30 / 74)$ had concomitant pelvic fracture diagnoses. Of these patients, eighteen $(60 \%, 18 / 30)$ underwent laparotomy only, while the other twelve patients (40\%, 12/30) required post-laparotomy TAE. Compared with the patients who underwent laparotomy only, more patients with Tile $\mathrm{B}_{1}$-type pelvic fractures (58.3\% vs. $11.1 \%$, $p=0.013$ ) required post-laparotomy TAE.

Conclusion: Regarding BAT patient management, the likelihood of post-laparotomy TAE should be considered in patients with concomitant pelvic fractures. Furthermore, more attention should be directed toward patients with Tile $\mathrm{B}_{1}$-type pelvic fractures because of the specific fracture pattern and impaction force.

Keyword: Pelvic fracture, Laparotomy, Transcatheter arterial embolization, Tile $B_{1}$

\section{Introduction}

Intraperitoneal and retroperitoneal hemorrhages may occur simultaneously in patients with concomitant blunt abdominal trauma (BAT) and unstable hemodynamics [1-3]. Additionally, BAT management principles have evolved to a non-operative management approach because of advancements in treatment concepts and improvements in

\footnotetext{
* Correspondence: drfu5564@yahoo.com.tw

${ }^{\dagger}$ Equal contributors

Department of Trauma and Emergency Surgery, Chang Gung Memorial

Hospital, Chang Gung University, 5, Fu-Hsing Street, Kwei Shan Township, Taoyuan, Taiwan
}

diagnostic modalities $[4,5]$. however, laparotomies are still necessary in some situations. In contrast, transcatheter arterial embolization (TAE) serves as an effective surgery alternative for retroperitoneal hemorrhage management [6-8]. Therefore, in addition to aggressive resuscitation, either a laparotomy or a TAE is usually needed for the hemostasis management of these patients. Furthermore, some complicated cases require both procedures; however, only limited information is available for hemorrhage origin assessment when only clinical presentation and primary tools are used for the evaluations. Although sonographic examination is utilized in emergency departments (ED)

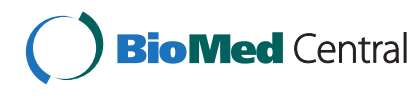

(c) 2015 Wu et al.; licensee BioMed Central. This is an Open Access article distributed under the terms of the Creative Commons Attribution License (http://creativecommons.org/licenses/by/4.0), which permits unrestricted use, distribution, and reproduction in any medium, provided the original work is properly credited. The Creative Commons Public Domain Dedication waiver (http://creativecommons.org/publicdomain/zero/1.0/) applies to the data made available in this article unless otherwise stated. 
worldwide, it can only detect intraperitoneal hemorrhage and has limitations regarding retroperitoneal hemorrhage surveillence [9-11]. Therefore, it is difficult to identify patients with concomitant intraperitoneal and retroperitoneal hemorrhages during primary evaluations over a short time period.

Patients usually undergo emergency laparotomies because of concomitant unstable hemodynamics and positive sonographic examination results $[9,10,12]$. However, if associated retroperitoneal hemorrhages are found intraoperatively and cannot be controlled surgically, then post-laparotomy TAE is required. The preparation of an angioembolization suite and the gathering of personnel are usually time-consuming, which may delay definitive hemostasis. Furthermore, transporting these patients between the operation and angiographic rooms is risky under such critical conditions. Therefore, the early identification of these patients is important to ensure that subsequent treatments can be initiated in a timely manner. Additionally, more information may be needed for physicians to predict retroperitoneal hemorrhages and further hemostatic procedure requirements.

In this study, we describe a retrospective observation of the management of patients with concomitant BAT and unstable hemodynamics. The different clinical course characteristics were delineated and compared, and the patients who underwent post-laparotomy TAE were investigated and analyzed. We attempted to determine the risk factors that indicated post-laparotomy TAE and analyzed the critical decision-making processes that occurred when only limited information was available.

\section{Materials and methods}

From May 2008 to October 2013, patients with concomitant BAT and unstable hemodynamic were retrospectively analyzed. They were evaluated and treated according to our established algorithm, which is based on the Advanced Trauma Life Support (ATLS) guidelines [13] (Figure 1). The Pelvic X-ray was routinely used as an adjunct to the primary survey in these patients. Unstable hemodynamics were defined as having systolic blood pressure $(\mathrm{SBP})<90 \mathrm{mmHg}$, without response to $2000 \mathrm{ml}$ of fluid resuscitation. Patients who died in the ED without undergoing other evaluations were not enrolled in the current study. The pelvic circumferential compression device (PCCD) was applied in the ED while the pelvic fracture diagnosed. Then the patients underwent emergency laparotomy and had positive sonographic examination results, which indicated intra-abdominal hemorrhage. Post-laparotomy TAE was indicated for the patients with retroperitoneal hemorrhages that were found intraoperatively. They were moved to an angiographic room after a damage-control laparotomy after intraperitoneal/retroperitoneal packing directly without additional computed

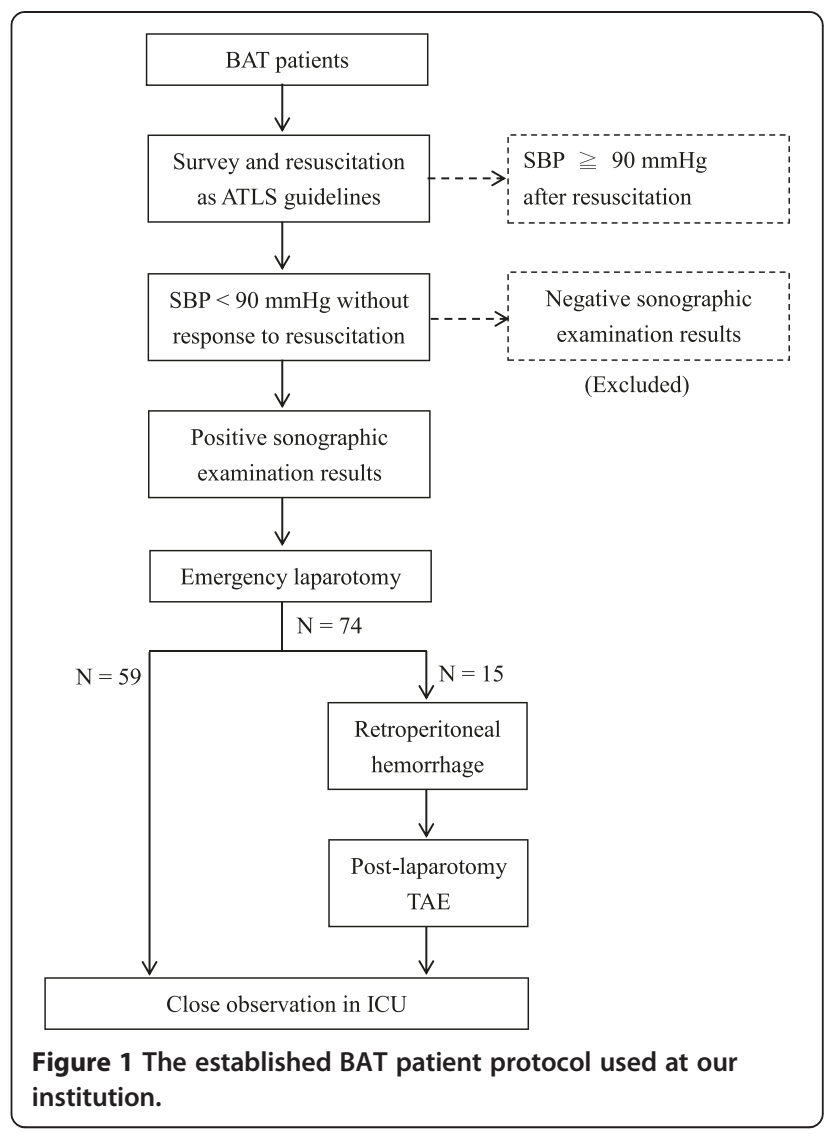

tomographic (CT) scan. These patients were taken to the intensive care unit (ICU) for further observation after these hemostasis procedures. In our institution, the operating and angiography rooms were available 24 hours per day, and a TAE could be performed within one hour.

In this study, the characteristics of the patients who required post-laparotomy TAEs were noted and investigated. Patient demographics, Revised Trauma Scores (RTSs), Injury Severity Scores (ISSs) and number of blood transfusions in the ED were routinely recorded. The Tile classification system was used to evaluate the pelvic fracture patterns $[14,15]$. The pelvic ring has a stable fracture with intact sacroiliac complex in type A injuries. The type $B$ and $C$ injuries were classified as unstable pelvic fracture $\left(B_{1}\right.$ : external rotational unstable, $\mathrm{B}_{2}$ : internal rotational unstable, $\mathrm{C}$ : both rotational and vertical unstable). The patients who underwent laparotomy only or laparotomy plus post-laparotomy TAE were compared.

All data are presented as patient percentages or means with standard deviations. The numerical data were compared using the Wilcoxon two-sample exact test. Nominal data were compared using Fisher's exact test. Multivariate logistic regression analysis was performed to determine the independent risk factors related to the 
requirement for post-laparotomy TAE. All statistical analyses were performed using the SPSS computer software package (version 13.0, Chicago, IL, USA). A value of $p<0.05$ was considered to be significant.

\section{Results}

During the 68-month study period, 3,871 patients with BAT diagnoses were sent to our ED. Thirty-nine patients died in the ED without undergoing further evaluations and were excluded. A total of 74 patients with concomitant BAT and unstable hemodynamics without response to $2000 \mathrm{ml}$ of fluid resuscitation were enrolled in this study. The mean patient age was 42.0 years (48 [64.7\%] males and 26 [35.3\%] females).

The various clinical course distributions for the patients are shown in Figure 1. Fifty-nine (79.7\%) patients underwent laparotomy only to treat intra-abdominal hemorrhage, and fifteen (20.3\%) patients underwent post-laparotomy TAE because of concomitant retroperitoneal hemorrhage. Table 1 compares the patients who underwent laparotomy only with patients who required post-laparotomy TAE. Pelvic fracture was present in $80.0 \%$ of the patients who required post-laparotomy TAE, which was significantly greater than the percentage of the patients who underwent laparotomy only $(80.0 \%$ vs. $30.5 \%, p<0.001)$. These patients had significantly higher ISSs $(24.6 \pm 24.1$ vs. $19.5 \pm 20.7, p=0.004)$, lower RTSs $(4.819 \pm 1.335$ vs. $6.007 \pm 0.772, p=0.011)$ and a greater amount of transfused blood $(1356.3 \pm 977.6 \mathrm{ml}$

\begin{tabular}{|c|c|c|c|}
\hline Variables & $\begin{array}{l}\text { Laparotomy only } \\
(\mathrm{N}=59)\end{array}$ & $\begin{array}{l}\text { Laparotomy } \rightarrow \text { TAE } \\
\quad(\mathrm{N}=15)\end{array}$ & $p$-value \\
\hline$\overline{\text { Age }}$ & $42.3 \pm 24.2$ & $40.6 \pm 23.1$ & $0.916^{\#}$ \\
\hline Gender (N) & & & $0.367^{\$}$ \\
\hline Female & $19(32.2 \%)$ & 7 (46.7\%) & \\
\hline Male & $40(67.8 \%)$ & $8(53.3 \%)$ & \\
\hline ISS & $19.5 \pm 20.7$ & $24.6 \pm 24.1$ & $0.004^{\#}$ \\
\hline RTS & $6.007 \pm 0.772$ & $4.819 \pm 1.335$ & $0.011^{\#}$ \\
\hline $\begin{array}{l}\text { Blood } \\
\text { transfusion (ml) }\end{array}$ & $887.3 \pm 934.9$ & $1356.3 \pm 977.6$ & $0.028^{\#}$ \\
\hline Pelvic fracture $(\mathrm{N})$ & & & $<0.001^{\$}$ \\
\hline Yes & $18(30.5 \%)$ & $12(80.0 \%)$ & \\
\hline No & $41(69.5 \%)$ & $3(20.0 \%)$ & \\
\hline Pelvis stability & & & $<0.001^{\$}$ \\
\hline Stable (tile A) & 55 (93.2\%) & $6(40.0 \%)$ & \\
\hline Unstable (tile B/C) & $4(6.8 \%)$ & $9(60.0 \%)$ & \\
\hline
\end{tabular}

The variables are expressed as means \pm SD.

"Wilcoxon two-sample exact test, ${ }^{\$}$ Fisher's exact test.
Table 2 The factors independently associated with post-laparotomy TAE in the overall patient population

\begin{tabular}{lcc}
\hline Variable & Odds ratio $(\mathbf{9 5} \% \mathrm{Cl})$ & $\boldsymbol{p}$-value \\
\hline Pelvic fracture & $3.4(2.2 \sim 11.4)$ & 0.018 \\
Blood transfusion $\geqq 1500 \mathrm{ml}$ & $8.7(0.7 \sim 15.2)$ & 0.302 \\
RTS $<5.5$ & $5.2(0.1 \sim 13.4)$ & 0.272 \\
ISS $\geqq 16$ & $2.2(1.6 \sim 9.5)$ & 0.048 \\
\hline
\end{tabular}

Multivariate logistic regression

vs. $887.3 \pm 934.9 \mathrm{ml}, p=0.028$ ) (Table 1$)$. Multivariate logistic regression analyses revealed that the presence of a pelvic fracture (odds ratio $[\mathrm{OR}]=3.4, p=0.018$ ) and an ISS $\geqq 16(\mathrm{OR}=2.2, p=0.048)$ were two significant predictive factors for patients requiring post-laparotomy TAE (Table 2).

In the current study, there were 30 concomitant pelvic fracture diagnoses $(40.5 \%, 30 / 74)$. Of these patients, eighteen $(60 \%, 18 / 30)$ underwent laparotomy only, while the other twelve patients $(40 \%, 12 / 30)$ required postlaparotomy TAE. Compared with the laparotomy-only patients, the post-laparotomy TAE patients required more transfused blood $(1542.8 \pm 1022.5 \mathrm{ml}$ vs. $914.5 \pm 425.9 \mathrm{ml}$, $p<0.001)$. Additionally, there were more patients with type $\mathrm{B}_{1}$ pelvic fractures $(58.3 \%$ vs. $11.1 \%, p=0.013)$ or unstable pelvic fractures $(75.0 \%$ vs. $22.2 \%, p=0.008)$ among these patients. Furthermore, the post-laparotomy TAE patients also demonstrated significantly higher ISSs $(26.3 \pm 14.1$ vs. $22.5 \pm 20.7, p=0.004)$ and lower RTSs $(4.115 \pm 2.431$ vs. $5.981 \pm 3.212, p=0.011)$ than the

Table 3 Comparisons between the pelvic fracture patients who underwent laparotomy only and the patients who required post-laparotomy TAE

\begin{tabular}{|c|c|c|c|}
\hline Variables & $\begin{array}{l}\text { Laparotomy only } \\
(\mathrm{N}=18)\end{array}$ & $\begin{array}{l}\text { Laparotomy } \rightarrow \text { TAE } \\
(\mathrm{N}=12)\end{array}$ & $p$-value \\
\hline Age & $41.8 \pm 16.7$ & $43.0 \pm 22.6$ & $0.854^{\#}$ \\
\hline Gender (N) & & & $1.000^{\$}$ \\
\hline Female & $5(27.8 \%)$ & $4(33.3 \%)$ & \\
\hline Male & $13(72.2 \%)$ & $8(66.7 \%)$ & \\
\hline ISS & $22.5 \pm 20.7$ & $26.3 \pm 14.1$ & $0.004^{\#}$ \\
\hline RTS & $5.981 \pm 3.212$ & $4.115 \pm 2.431$ & $0.011^{\#}$ \\
\hline $\begin{array}{l}\text { Blood } \\
\text { transfusion (ml) }\end{array}$ & $914.5 \pm 425.9$ & $1542.8 \pm 1022.5$ & $<0.001^{\#}$ \\
\hline Fracture pattern (N) & & & $0.013^{\$}$ \\
\hline Tile $B_{1}$ & $2(11.1 \%)$ & $7(58.3 \%)$ & \\
\hline $\begin{array}{l}\text { Non-Tile } B_{1} \\
\left(A+B_{2}+C\right)\end{array}$ & $16(88.9 \%)$ & $5(41.7 \%)$ & \\
\hline Pelvis stability & & & $0.008^{\$}$ \\
\hline Stable (tile A) & $14(77.8 \%)$ & $3(25.0 \%)$ & \\
\hline Unstable (tile B/C) & $4(22.2 \%)$ & $9(75.0 \%)$ & \\
\hline
\end{tabular}

Variables are expressed as means \pm SD.

"Wilcoxon two-sample exact test, ${ }^{\$}$ Fisher's exact test. 
Table 4 The factors independently associated with post-laparotomy TAE in pelvic fracture patients

\begin{tabular}{lcc}
\hline Variable & Odds ratio $(\mathbf{9 5 \%}$ Cl) & $\boldsymbol{p}$-value \\
\hline Tile $\mathrm{B}_{1}$ pelvic fracture & $6.4(4.0 \sim 15.1)$ & 0.002 \\
Blood transfusion $\geqq 1500 \mathrm{ml}$ & $2.9(0.1 \sim 4.6)$ & 0.519 \\
RTS $<5.5$ & $3.3(0.4 \sim 5.1)$ & 0.238 \\
ISS $\geqq 16$ & $5.5(3.6 \sim 14.2)$ & 0.001 \\
\hline
\end{tabular}

Multivariate logistic regression.

other patients (Table 3 ). Table 4 shows that a type $B_{1}$ pelvic fracture $(\mathrm{OR}=6.4, p=0.002)$ and an ISS $>16$ $(\mathrm{OR}=5.9, p=0.002)$ were two independent risk factors for post-TAE laparotomy.

In current study, there were three patients without pelvic fractures who required post-laparotomy TAE. Their angiographic examinations revealed that all of them had active lumbar arterial hemorrhage.

\section{Discussion}

Blunt abdominal trauma may result in intraperitoneal or retroperitoneal hemorrhages, which are both potentially life-threatening and require organized rapid evaluation and treatment [1-3]. An emergency laparotomy is usually indicated for an intraperitoneal hemorrhage that presents as intra-abdominal free fluid during sonography. In the management of the associated retroperitoneal hemorrhage, the PCCD and even the extraperitoneal packing are required to decrease the retroperitoneal hemorrhage before the laparotomy. Then the further TAE could be performed for the definitive hemostasis. However, in these patients, it is difficult to consider the requirement of TAE until persistent expansion of a retroperitoneal hematoma was detected intraoperatively. At that time, the TAE preparation would be initiated. After a damage-control laparotomy, with intraperitoneal/retroperitoneal packing, the patients would be sent to an angiographic room. Therefore, they would remain in critical condition with an ongoing hemorrhage while waiting for an angioembolization suite to be prepared and for personnel to be gathered.

In the current study, 74 patients underwent emergency laparotomy because of concomitant unstable hemodynamics and positive sonographic examination results. Retroperitoneal hemorrhage was found intraoperatively in 15 of these patients (20.3\%); thus, they underwent post-laparotomy TAE. The percentage of these patients with concomitant pelvic fracture was significantly greater than that of the patients who underwent laparotomy only $(80.0 \%$ vs. $30.5 \%$, $p<0.001)$. Furthermore, multivariate logistic regression analyses revealed that the presence of a pelvic fracture was a significant factor in predicting the need for post-laparotomy TAE in such patients $(\mathrm{OR}=3.4, p=0.018)$ (Table 2). Pelvic fractures usually stem from high-kinetic-energy blunt trauma and can result in retroperitoneal hemorrhage. Associated hemodynamic instability was reported in 5-20\% of these patients, and the subsequent reported mortality rate was $18-40 \%$ [16-20]. Therefore, in addition to intraperitoneal hemorrhage, an associated retroperitoneal hemorrhage should also be considered when managing patients with BAT and unstable hemodynamics with concomitant pelvic fractures.

Previous reports have proposed that unstable pelvic fractures indicate major ligamentous disruptions that are often associated with life threatening arterial bleeding $[14,15,21]$. Additionally, pelvic fracture patterns were classified into three main groups according to the integrity of the posterior sacroiliac complex by Tile $[14,15]$. The focused analysis of pelvic fracture patients in the current study revealed that the patients who required post-laparotomy TAE had a significantly greater percentage of unstable pelvises $(75.0 \%$ vs. $22.2 \%, p=0.008)$ and type $B_{1}$ (external rotational unstable) pelvic fractures

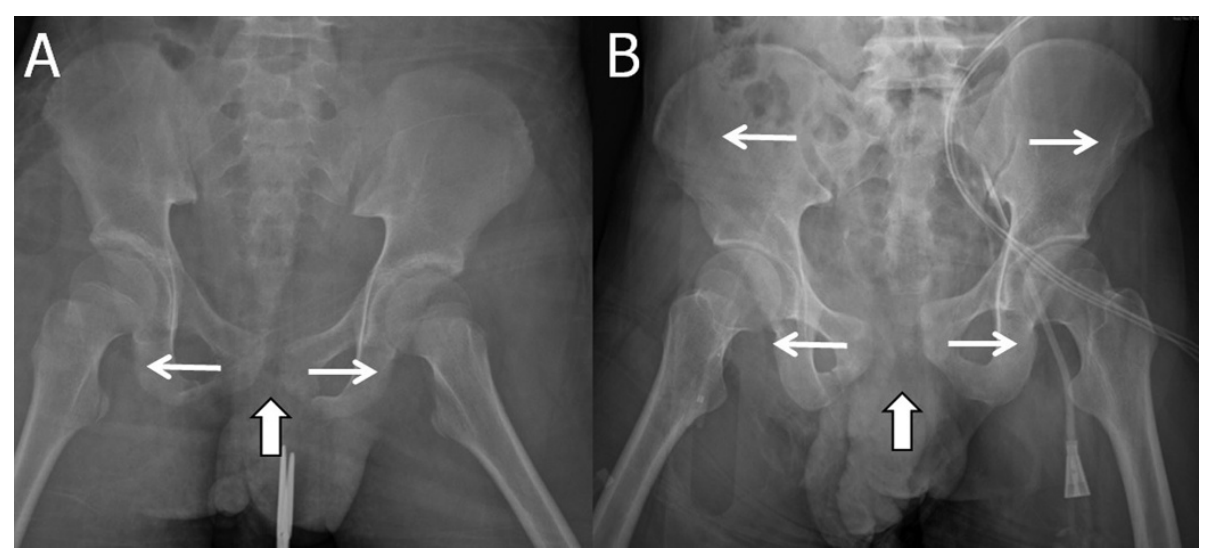

Figure 2 Plain external rotational type pelvic fracture films. The large arrows indicate the disruptive force direction and side of the impact, and the small arrows indicate the splaying of the pubic symphysis (A) and further external iliac wing rotation (B). 
(58.3\% vs. $11.1 \%, p=0.013)$ compared with the patients who underwent laparotomy only (Table 3). Furthermore, multiple logistic regression analyses in the current study also revealed that type $B_{1}$ pelvic fractures were an independent risk factor for concomitant intraperitoneal and retroperitoneal hemorrhages, with approximately an eight-fold increased risk of requiring post-laparotomy TAE $(\mathrm{OR}=6.4, p=0.002)$ (Table 4).

Unlike other pelvic fractures, type $B_{1}$ pelvic fractures create complete anterior pelvis diastases and partial or complete posterior pelvis diastases (Figure 2) [15,18,22]. The tensile and shearing force of such injuries are generally from the middle and front. They tend to open the anterior pelvis, which is thinner than the iliac bone, over the lateral side by pubic ramus fracture or pubic symphysis ligaments rupture mechanisms (Figure 2A). Furthermore, additional external rotational forces may result in splaying of the anterior pelvis and external rotation of the iliac wings, which provide circumferential protection of the intra-abdominal organs (Figure 2B). Therefore, it is reasonable that such impaction, with relative weak protection, may be associated with a greater risk of intraperitoneal injury. Additionally, it was reported that the external rotational pelvic fracture involves greater vessel disruption and more hemorrhaging than other injury mechanisms [22]. Our previous report also indicated its association with bilateral sacroiliac joint injuries and further bilateral arterial injuries [23]. According to the above theories, concomitant intraperitoneal and retroperitoneal hemorrhages may occur in type $B_{1}$ pelvic fracture patients. In managing patients with unstable hemodynamics and positive sonographic examinations, associated retroperitoneal hemorrhages should be suspected in patients with concomitant type $B_{1}$ pelvic fractures.

Another concern is in evaluating other associated injuries. The ISS has been used to evaluate the severity of other associated injuries $[24,25]$. This trauma scoring system is a tool that can assist with the triage, prognosis prediction and resource allocation of ED trauma patients. In the current study, the post-laparotomy TAE patients also had significantly higher ISSs than the patients who underwent laparotomy only. Multiple logistic regression analysis also revealed that a higher ISS $(\geq 16)$ was independently associated with an increased risk for post-laparotomy TAE. Although the precise ISS was not available in the ED, the current study results indicate that other associated injuries may increase the risk of subsequent deterioration requiring additional procedures.

The early identification of post-laparotomy TAE patients might shorten the waiting time for TAE. Furthermore, the risk that occurs while patients wait and during transportation could also be reduced. Pelvic fractures can easily be diagnosed on a plain pelvis film; thus, type $B_{1}$ pelvic fractures could also be identified accordingly. This fact revealed that concomitant retroperitoneal hemorrhages and the necessity of additional hemostatic procedures for unstable patients could be surmised with primary tool evaluations (e.g., sonographic examinations and plain pelvis films). To save additional time, one suggestion is to simultaneously prepare for TAE while performing laparotomy in type $B_{1}$ pelvic fracture patients. This process may diminish the increased risk of injuries associated with concomitant retroperitoneal hemorrhages, which may require post-laparotomy TAE.

The limitations of this study are its retrospective nature and small number of examined cases. Our conclusions may be limited by a possible selection bias. Additionally, the role of CT scan was not discussed in the current study. We agree that CT scan could augment the detection of soft tissue injuries and active arterial bleeding; however, CT scans are not recommended for most patients with unstable hemodynamics [13]. Further studies with larger sample sizes and prospective designs are needed to establish precise treatment plan algorithms in the ED.

\section{Conclusion}

In BAT patient management, the likelihood of postlaparotomy TAE should be considered in concomitant pelvic fracture patients. Furthermore, more attention should be directed toward patients with Tile $\mathrm{B}_{1}$-type pelvic fractures because of its specific fracture pattern and impaction force.

\section{Competing interests}

The authors declare that they have no competing interests.

\section{Authors' contributions}

CYW: Article writing, data collection. SJY: Article writing, data collection, statistic analysis. CYF: Supervisor of article writing. CHL, SC K, YPH, BCL, KCY, SYW: Data collection. All authors read and approved the final manuscript.

\section{Authors' information}

Chun-Yi Wu and Shang-Ju Yang are first author.

Received: 16 August 2014 Accepted: 16 January 2015 Published: 27 January 2015

\section{References}

1. Fang J-F, Shih L-Y, Wong Y-C, Lin B-C, Hsu Y-P. Angioembolization and laparotomy for patients with concomitant pelvic arterial hemorrhage and blunt abdominal trauma. Langenbecks Arch Surg. 2011;396:243-50.

2. Fang J-F, Wong Y-C, B- L, Hsu Y-P, Chen M-F. Usefulness of multidetector computed tomography for the initial assessment of blunt abdominal trauma patients. World J Surg. 2006;30:176-82.

3. Tyburski JG, Wilson RF, Dente C, Steffes C, Carlin AM. Factors affecting mortality rates in patients with abdominal vascular injuries. J Trauma. 2001;50:1020-6. NOM.

4. Raza M, Abbas Y, Devi V, Prasad KV, Rizk KN, Nair PP. Non operative management of abdominal trauma - a 10 years review. World J Emerg Surg. 2013;8:14.

5. Sriussadaporn S, Pak-art R, Tharavej C, Sirichindakul B, Chiamananthapong S. A multidisciplinary approach in the management of hepatic injuries. Injury. 2002;33:309-15.

6. Hauschild O, Aghayev E, von Heyden J, Strohm PC, Culemann U, Pohlemann T, et al. Angioembolization for pelvic hemorrhage control: results from the German pelvic injury register. J Trauma Acute Care Surg. 2012;73:679-84. 
7. Jeske HC, Larndorfer R, Krappinger D, Attal R, Klingensmith M, Lottersberger C, et al. Management of hemorrhage in severe pelvic injuries. J Trauma. 2010;68:415-20.

8. Miller PR, Moore PS, Mansell E, Meredith JW, Chang MC. External fixation or arteriogram in bleeding pelvic fracture: initial therapy guided by markers of arterial hemorrhage. J Trauma. 2003;54:437-43.

9. Verbeek DO, Zijlstra IA, van der Leij C, Ponsen KJ, van Delden OM, Goslings JC. The utility of FAST for initial abdominal screening of major pelvic fracture patients. World J Surg. 2014;38:1719-25.

10. Walcher F, Weinlich M, Conrad G, Schweigkofler U, Breitkreutz R, Kirschning $T$, et al. Prehospital ultrasound imaging improves management of abdominal trauma. Br J Surg. 2006;93:238-42.

11. Tayal VS, Nielsen A, Jones AE, Thomason MH, Kellam J, Norton HJ. Accuracy of trauma ultrasound in major pelvic injury. J Trauma. 2006;61:1453-7.

12. Clarke JR, Trooskin SZ, Doshi PJ, Greenwald L, Mode CJ. Time to laparotomy for intra-abdominal bleeding from trauma does affect survival for delays up to 90 min. J Trauma. 2002;52:420-5.

13. Chapleau W, Al-Khatib J, Haskin D, LeBlanc P, Cardenas G, Borum S, et al. Advanced trauma life support. 9th ed. Chicago, IL: ATLS Subcommittee; American College of Surgeons' Committee on Trauma; International ATLS working group; 2013. J Trauma Acute Care Surg.

14. Pennal GF, Tile M, Waddell JP, Garside H. Pelvic disruption: assessment and classification. Clin Orthop Relat Res. 1980;151:12-21.

15. McMurtry R, Walton D, Dickinson D, Kellam J, Tile M. Pelvic disruption in the polytraumatized patient: a management protocol. Clin Orthop Relat Res. 1980;151:22-30.

16. Starr AJ, Griffin DR, Reinert CM, Frawley WH, Walker J, Whitlock SN, et al. Pelvic ring disruptions: prediction of associated injuries, transfusion requirement, pelvic arteriography, complications, and mortality. J Orthop Trauma. 2002;16:553-61.

17. Smith W, Williams A, Agudelo J, Shannon M, Morgan S, Stahel P, et al. Early predictors of mortality in hemodynamically unstable pelvis fractures. J Orthop Trauma. 2007;21:31-7.

18. Cryer HM, Miller FB, Evers BM, Rouben LR, Seligson DL. Pelvic fracture classification: correlation with hemorrhage. J Trauma. 1988;28:973-80.

19. Brun J, Guillot S, Bouzat P, Broux C, Thony F, Genty C, et al. Detecting active pelvic arterial haemorrhage on admission following serious pelvic fracture in multiple trauma patients. Injury. 2014;45:101-6.

20. Biffl WL, Smith WR, Moore EE, Gonzalez RJ, Morgan SJ, Hennessey T, et al. Evolution of a multidisciplinary clinical pathway for the management of unstable patients with pelvic fracture. Ann Surg. 2001;233:843-50.

21. Fu CY, Wu SC, Chen RJ, Wang YC, Chung PK, Yeh CC, et al. Evaluation of pelvic fracture stability and the need for angioembolization: pelvic instabilities on plain film have an increased probability of requiring angioembolization. Am J Emerg Med. 2009;27:792-6.

22. Dalal SA, Burgess AR, Siegel JH, Young JW, Brumback RJ, Poka A, et al. Pelvic fracture in multiple trauma: classification by mechanism is key to pattern of organ injury, resuscitative requirements, and outcome. J Trauma. 1989:29:981-1000.

23. Fu CY, Hsieh CH, Wu SC, Chen RJ, Wang YC, Shih CH, et al. Anterior-posterior compression pelvic fracture increases the probability of requirement of bilateral embolization. Am J Emerg Med. 2013;31:42-9.

24. Sacco WJ, MacKenzie EJ, Champion HR, Davis EG, Buckman RF. Comparison of alternative methods for assessing injury severity based on anatomic descriptors. J Trauma. 1999:47:441-6.

25. Baker SP, O'Neill B, Haddon Jr W, Long WB. The injury severity score: a method for describing patients with multiple injuries and evaluating emergency care. J Trauma. 1974;14:187-96.

doi:10.1186/1749-7922-10-4

Cite this article as: Wu et al.: The risk factors of concomitant intraperitoneal and retroperitoneal hemorrhage in the patients with blunt abdominal trauma. World Journal of Emergency Surgery 2015 10:4 